[0212-7199 (2003) 20: 6; pp 279-281] ANALES DE MEDICINA INTERN Copyright $\odot 2003$ ARAN EDICIONES, S.L.

AN. MED. INTERNA (Madrid) Vol. 20, N. ${ }^{\circ} 6$, pp. $279-281,2003$

\title{
Papel actual de los nuevos calcioantagonistas dihidropiridínicos en el tratamiento de la hipertensión arterial
}

Coca A. Papel actual de los nuevos calcioantagonistas dihidropiridinicos en el tratamiento de la hipertensión arterial. An Med Interna (Madrid) 2003; 20: 279-281.

El beneficio potencial del tratamiento antihipertensivo no reside, en sí mismo, en el descenso de las cifras de presión arterial sino en la capacidad de reducir la probabilidad de padecer un episodio cerebrovascular, cardiovascular o renal, sea o no mortal, en un hipertenso concreto o en un grupo de estos pacientes, mediante el descenso de las cifras de presión arterial. Para conseguir tal descenso disponemos de seis grupos de fármacos antihipertensivos, uno de los cuales es el constituido por los llamados calcioantagonistas, antagonistas del calcio o bloqueantes de los canales de calcio. Se trata de un grupo heterogéneo de fármacos que bloquean los canales de calcio cuya apertura está regulada por diferencias de potencial eléctrico, los llamados canales "L" o potencial-dependiente. Tres subtipos están disponibles para su uso clínico: las dihidropiridinas (nifedipino, amlodipino, barnidipino, manidipino, lercanidipino..), las fenilalquilaminas (verapamilo) y las benzotiazepinas (diltiazem). Su acción en la fibra muscular lisa arteriolar disminuye la concentración de calcio libre citosólico, con lo que desciende el tono vasomotor y la resistencia periférica. El efecto predominante es la vasodilatación arteriolar, particularmente notable en las dihidropiridinas, por lo que reducen las cifras de presión arterial y aumentan el flujo cerebral y coronario. Por su particular mecanismo de acción se han venido utilizando ampliamente en el tratamiento de la hipertensión arterial.

Existen múltiples calcioantagonistas de liberación sostenida o vida media prolongada, entre los que lercanidipino es un buen exponente, que ejercen el efecto antihipertensivo a lo largo de todo el intervalo de 24 horas, se administran en dosis única diaria y mejoran el cumplimiento terapéutico por los pacientes, son bien tolerados, se pueden asociar con otros antihipertensivos, no presentan contraindicaciones formales, se pueden utilizar en patologías asociadas (procesos osteoarticulares degenerativos, insuficiencia respiratoria, diabetes mellitus o gota úrica), tienen un efecto neutro sobre el perfil metabólico y no alteran, o mejoran, la función renal y la hemodinámica cardiovascular. A todo ello hay que añadir los datos muy recientes que demuestran su capacidad de reducir la mortalidad y morbilidad en prevención primaria de la hipertensión arterial, lo que les confiere el papel de fármacos de primera línea, sea en monoterapia o en asociación, en el tratamiento de la hipertensión arterial (1-3).

En este mismo número de la revista se publican dos artículos originales correspondientes a los estudios LAPSE (4) y LERCAPSICO (5), ambos realizados en el ámbito de la Atención Primaria. El primero es un estudio de eficacia y seguridad mientras que el segundo analiza aspectos relacionados con la calidad de vida de los pacientes tratados con el nuevo calcioantagonista dihidropiridínico, lercanidipino. En ambos estudios se muestra no sólo su eficacia antihipertensiva y su excelente tolerabilidad, sino también el positivo perfil metabólico del fármaco, a lo que se añade el impacto sobre la calidad de vida observado en el estudio LERCAPSICO.

El perfil metabólico es un aspecto relevante de los fármacos antihipertensivos y en todos los ensayos clínicos controlados realizados hasta la actualidad se ha podido demostrar que los calcioantagonistas tienen efectos neutros sobre el metabolismo hidrocarbonado y lipídico. Por tanto, en los hipertensos con hiperlipidemias, y en los hipertensos con diabetes tipo 2 cuyo tratamiento inicial con antagonistas de los receptores de angiotensina II $(6,7)$ (ARA II) o con inhibidores de la enzima conversora de la angiotensina (8) (IECA) no consiga reducir la PA por debajo de 130/80 mmHg, los fármacos de elección para asociar a los primeros son los calcioantagonistas.

Respecto al efecto antiaterogénico de los calcioantagonistas disponemos ya de diversas evidencias positivas en estudios clínicos a largo plazo (9-12). Previamente, algunas observaciones experimentales habían sugerido que los calcioantagonistas eran capaces de inhibir la migración y proliferación de células musculares lisas en cultivo, así como de retrasar el desarrollo y evolución de la placa de ateroma en animales sometidos a dietas ricas en colesterol (13). Sin embargo, la evidencia de que este efecto antiaterogénico experimental se podía reproducir en la clínica era muy débil. En el estudio INTACT (14) el tratamiento con nifedipino de vida media corta, administrado a dosis elevadas durante tres años en pacientes afectos de ateromatosis coronaria comprobada angiográficamente, no consiguió disminuir significativamente el número y gravedad de las lesiones coronarias ya establecidas en comparación con el placebo. No obstante, el 
número de nuevas lesiones por paciente sí fue inferior al que se observó en los que recibieron placebo, con una reducción significativa del $28 \%$. En este mismo sentido, un estudio italiano demostró que los pacientes con lesiones angiográficas coronarias tratados con nifedipino mostraban una menor progresión de las lesiones respecto a los tratados con propranolol o dinitrato de isosorbide (15).

Es posible que la falta de confirmación clínica inequívoca de las expectativas generadas por la investigación experimental estuviera relacionada con la utilización en aquellos estudios de calcioantagonistas de vida media corta (14). En efecto, la estimulación simpática refleja como mecanismo contrarregulador de la vasodilatación arteriolar y el correspondiente aumento de catecolaminas circulantes, podría haber contrarrestado el beneficio del bloqueo de los canales de calcio sobre el crecimiento de la placa de ateroma. En la actualidad disponemos de los resultados de diversos ensayos clínicos multinacionales con calcioantagonistas de larga duración de acción, que no promueven hiperactivación simpática refleja, que han demostrado su capacidad de prevenir la progresión del grosor de la íntima-media (GIM) carotídea de los hipertensos (9-11), GIM que sí aumenta a pesar de la correcta reducción de presión en los pacientes tratados con diuréticos (11).

Por tanto, los calcioantagonistas de acción prolongada han demostrado con absoluta solvencia efectos beneficiosos. En prevención secundaria, el estudio PRAISE demostró mejoría de la supervivencia de pacientes con insuficiencia cardiaca de etiología no isquémica con amlodipino (16). El estudio DAVIT-II con verapamilo (17) y el MDPIT con diltiazem (18) también demostraron efectos beneficiosos en la prevención secundaria de episodios coronarios en ausencia de insuficiencia cardiaca. En la prevención primaria de HTA los datos definitivos del beneficio del tratamiento calcioantagonista son muy recientes. Hoy día está perfectamente demos- trado que el tratamiento calcioantagonista disminuye la tasa de AVC en pacientes ancianos con HTA sistólica aislada (19) y de enfermedad coronaria en pacientes con HTA en los que se reduce la PA hasta $80 \mathrm{mmHg}$ (20). Los estudios STOP-2 (21), INSIGHT (1), NORDIL (22) y ALLHAT (2) que comparaban el grado de protección del tratamiento calcioantagonista con el tratamiento diurético y o con betabloqueantes en la HTA, muestran que los calcioantagonistas poseen un efecto protector similar al de otros tratamientos antihipertensivos, probablemente algo mayor por lo que respecta a la prevención de AVC $(23,24)$, similar en la enfermedad coronaria (3) y algo menor en la insuficiencia cardíaca congestiva $(23,24)$.

Por tanto, como recomienda la Food and Drug Administration (FDA), para el tratamiento de la HTA deben utilizarse únicamente los calcioantagonistas de liberación retardada o vida media larga. Estos fármacos no producen activación simpática refleja, controlan la presión arterial a lo largo de las 24 horas y evitan los picos matutinos de PA que se han relacionado con una mayor tasa de episodios cardiovasculares (25). En cualquier caso, la justificación del uso de calcioantagonistas como fármacos de primera elección en el tratamiento general de la HTA está avalado por sus efectos cardiovasculares, renales y metabólicos, claramente superiores a los de los fármacos de referencia del JNC-VI, así como por su efecto beneficioso en la prevención primaria y secundaria de morbilidad y mortalidad cardiovasculares.

\section{A. COCA}

\section{Unidad de Hipertensión. Hospital Clínico. Instituto de Investigaciones Biomédicas Augusto Pi Suñer (IDIBAPS). Universidad de Barcelona}

\section{Bibliografía}

1. Brown MJ, Palmer CR, Castaigne A, et al. Morbidity and mortality in patients randomised to double-blind treatment with a long-action calcium-channel blocker or diuretic in the International Nifedipine GITS study: Intervention as a Goal in Hypertension Treatment (INSIGHT). Lancet 2000; 356: 366-372.

2. Major outcomes in high-risk hypertensive patients randomized to angiotensin-converting enzyme inhibitor or calcium channel blocker vs diuretic. The Antihypertensive and Lipid-Lowering treatment to prevent Heart Attack Trial (ALLHAT). JAMA 2002; 288: 2981-2997.

3. Pepine CJ for the INVEST group. Main outcomes from randomized trial of a calcium antagonist vs noncalcium antagonist blood pressure treatment strategy in patients with coronary artery disease: the international verapamil-trandolapril study (INVEST). Lancet 2003;

4. Roberto-Robles N, Canelada JA, Iglesias M, Angulo E, López-Acedo A, Díaz-Olea E, et al. Evaluación del lercanidipino en Atención Primaria: seguridad y eficacia. Resultados del estudio LAPSE. An Med Interna (Madrid) 2003; 20: 282-286.

5. Abellán J, Martinez JF, Merino J, Gil V, Latorre J, Divisón JA, et al Valoración de la semiología psicosomática en hipertensos tratados con lercanidipino (Estudio LERCADIPSO). An Med Interna (Madrid) 2003; 20: 287-291.

6. Brenner BM, Cooper ME, de Zeeuw D, et al. Effects of losartan on renal and cardiovascular outcomes in patients with type 2 diabetes and nephropathy. N Engl J Med 2001; 345: 861-869.

7. Lewis EJ, Hunsicker LG, Clarke WR, Berl T, Pohl MA, Lewis JB, et al. Renoprotective effect of the angiotensin-receptor antagonist irbersartan in patients with nephropathy due to type 2 diabetes. N Engl J Med 2001; 345: 851-860.

8. Heart Outcomes Prevention Evaluation (HOPE) Study Investigators. Effects of ramipril on cardiovascular and microvascular outcomes in people with diabetes mellitus: results of the HOPE study and MICROHOPE substudy. Lancet 2000; 355: 253-259.

9. Pitt B, Byington R, Furberg CD, Hunninghake DB, Mancini J, Miller $\mathrm{ME}$, et al for the PREVENT Investigators. Effect of amlodipine on the progression of atherosclerosis and the occurrence of clinical events. Circulation 2000; 102: 1503-1510.

10. Simon A, Gariepy J, Moyse D, Levenson J. Differential effects of nifedipine and co-amilozide on the progression of early carotid wall changes. Circulation 2001; 103: 2949-2954.

11. Motro M, Shemesh J. Calcium channel blocker nifedipine slows down progression of coronary calcification in hypertensive patients compared with diuretics. Hypertension 2001; 37: 1410-1413.

12. Zanchetti A, Bond MG, Henning M, Neiss A, Mancia G, Dal Palu C, et al. Risk factors associated with alterations in carotid intima-media thickness in hypertension: baseline data from the European Lacidipine Study on Atherosclerosis. J Hypertens 1998; 16: 949-961.

13. Holzgreve H, Bürkle B. Anti-atherosclerotic effects of calcium antagonists. J Hypertens 1993; 11 (Suppl 1): s55-s59.

14. Lichtlen PR, Hugenholtz PG, Raffienbeul W, Hecker H, Jost S, Deckers JW, on behalf of the INTACT group. Retardation of angiographic progression of coronary artery disease by nifedipine: results of the International Nifedipine Trial on Antiatherosclerotic Therapy (INTACT). Lan- 
cet 1990; 335: 1109-1113.

15. Loaldi A, Polese A, Montorsi P, De Cesare N, Fabbiocchi F, Ravagnani $\mathrm{P}$, et al. Comparison of nifedipine, propranolol and isosorbide dinitrate on angiographic progression and regression of coronary arterial narrowings in angina pectoris. Am J Cardiol 1989; 64: 433 439.

16. Packer M, O'Connor CM, Ghali JK, et al. Effect of amlodipine on morbidity and mortality in severe chronic heart failure. Prospective Randomized Amlodipine Survival Evaluation Study Group. N Engl J Med 1996; 335: 1107-1114.

17. The Danish Study Group on Verapamil in Myocardial Infarction. Effect of verapamil on mortality and major events after acute myocardial infarction (The Danish Verapamil Infarction Trial DAVIT II). Am J Cardiol 1990; 66:779-785.

18. The Multicenter Diltiazem Postinfarction Trial Research Group. The effect of diltiazem on mortality and reinfarction after myocardial infarction. N Eng J Med 1988; 319: 385-392.

19. Staessen JA, Fagard R, Thijs L, et al. Randomised double-blind comparison of placebo and active treatment for older patients with isolated systolic hypertension. The Systolic Hypertension in Europe (Syst-Eur) Trial Investigators. Lancet 1997; 350: 757-764.

20. Hansson L, Zanchetti A, Carruthers SG, et al. Effects of intensive blood-pressure lowering and low-dose aspirin in patients with hypertension: principal results of the Hypertension Optimal Treatment (HOT) randomised trial. Lancet 1998; 351: 1755-1762.

21. Lindholm LH, Hansson L, Ekborn T, et al. Comparison of antihypertensive treatments in preventing cardiovascular events in elderly diabetic patients: Results from the Swedish Trial in Old Patients with Hypertension-2. STOP Hypertension-2 Study Group. J Hypertens 2000; 18: 1671-1675.

22. Hansson L, Hedner T, Lund-Johansen P, et al. Randomised trial of effects of calcium antagonists compared with diuretics and betablockers on cardiovascular morbidity and mortality in hypertension: The Nordic Diltiazem (NORDIL) study. Lancet 2000; 356: 359365 .

23. Blood Pressure Lowering Trialist Collaboration. Effects of ACE inhibitors, calcium antagonists, and other blood-pressure-lowering drugs: results of prospectively designed overviews of randomised trials. Lancet 2000; 355: 1955-1964.

24. Staessen JA, Wang JG, Thijs L. Cardiovascular protection and blood pressure reduction: a meta-analysis. Lancet 2001; 358: 1305-1315.

25. Frishman WH, Rosenberg A, Katz B. calcium antagonists in the management of systemic hypertension: the impact of sustained-release drugdelivery systems. Coronary Art Dis 1994; 5: 4-13. 